\title{
Strong monogamy conjecture for multiqubit entanglement: The four-qubit case
}

\author{
Bartosz Regula, ${ }^{1}$ Sara Di Martino, ${ }^{2,1}$ Soojoon Lee, ${ }^{3,1}$ and Gerardo Adesso ${ }^{1}$ \\ ${ }^{1}$ School of Mathematical Sciences, The University of Nottingham, \\ University Park, Nottingham NG7 2RD, United Kingdom \\ ${ }^{2}$ Dipartimento di Matematica, Università di Bari, I-70125 Bari, Italy \\ ${ }^{3}$ Department of Mathematics and Research Institute for Basic Sciences, Kyung Hee University, Seoul 130-701, Korea
}

(Dated: January 15, 2016)

\begin{abstract}
We investigate the distribution of bipartite and multipartite entanglement in multiqubit states. In particular we define a set of monogamy inequalities sharpening the conventional Coffman-Kundu-Wootters constraints, and we provide analytical proofs of their validity for relevant classes of states. We present extensive numerical evidence validating the conjectured strong monogamy inequalities for arbitrary pure states of four qubits.
\end{abstract}

PACS numbers: 03.67.Mn, 03.65.Ud

Introduction. - Entanglement is one of the most fundamental and intriguing features of quantum mechanics. It plays a crucial role for applications in quantum information and communication, and in a variety of areas ranging from quantum field theory to condensed matter, statistical physics, thermodynamics, and biology [1-3]. Despite its central importance, however, the physical understanding and mathematical description of its essential characteristics remain highly nontrivial tasks, particularly when many-particle systems are analyzed.

One of the key properties distinguishing entanglement from classical correlations is its monogamy: entanglement cannot be freely shared among multiple parties [4]. Monogamy is a consequence of the no-cloning theorem [5--7], and is obeyed by several types of nonclassical correlations, including Bell nonlocality [8], Einstein-Podolsky-Rosen steering [9] and contextuality [10], albeit not discord-type correlations [11].

In 2000 Coffman, Kundu, and Wootters (CKW) formalized the monogamy of entanglement for a three-qubit system in the form of a quantitative constraint, known as 'monogamy inequality' [12]. Given an arbitrary pure state $|\psi\rangle \in \mathcal{H}=$ $\left(\mathbb{C}^{2}\right)^{\otimes 3}$ of three qubits $q_{1}, q_{2}$, and $q_{3}$, the $\mathrm{CKW}$ inequality with respect to the choice of $q_{1}$ as a focus can be written as

$$
\tau_{q_{1} \mid\left(q_{2} q_{3}\right)}^{(1)}(|\psi\rangle) \geq \tau_{q_{1} \mid q_{2}}^{(2)}(|\psi\rangle)+\tau_{q_{1} \mid q_{3}}^{(2)}(|\psi\rangle) .
$$

Here $\tau_{q_{i} \mid q_{j}}^{(2)}$ denotes the bipartite entanglement in the reduced state of the pair of qubits $q_{i}$ and $q_{j}$, quantified by a computable entanglement monotone known as two-tangle, or simply tangle [12]14]. The term $\tau_{q_{1} \mid\left(q_{2} q_{3}\right)}^{(1)}$ denotes the one-tangle, a measure of entanglement between $q_{1}$ and the rest of the system, given by the linear entropy of the marginal state of qubit $q_{1}$,

$$
\tau_{q_{1} \mid\left(q_{2} q_{3}\right)}^{(1)}(|\psi\rangle)=4 \operatorname{det} \rho_{1},
$$

where $\rho_{1}=\operatorname{Tr}_{q_{2} q_{3}}(|\psi\rangle\langle\psi|)$ is the density matrix of qubit $q_{1}$ and $\operatorname{Tr}_{X}(\rho)$ indicates the partial trace of $\rho$ over subsystem $X$.

The meaning of Eq. (1) is clear: the entanglement between $q_{1}$ and the two other qubits taken as a group cannot be less than the sum of the individual entanglements between $q_{1}$ and each of the two remaining qubits. Similar inequalities can be written by selecting $q_{2}$ or $q_{3}$ as focus qubits. Remarkably, the

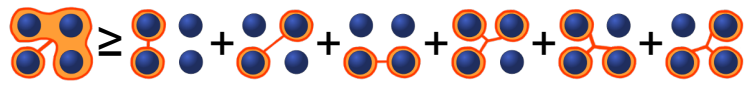

FIG. 1: Strong monogamy of four-qubit entanglement.

difference between left and right hand side of (1) can be interpreted as a quantifier of the entanglement genuinely shared among the three qubits. Precisely, one can define the residual three-qubit tangle_-or, in short, three-tangle_of $|\psi\rangle$ as

$$
\tau_{q_{1}\left|q_{2}\right| q_{3}}^{(3)}(|\psi\rangle):=\tau_{q_{1} \mid\left(q_{2} q_{3}\right)}^{(1)}(|\psi\rangle)-\tau_{q_{1} \mid q_{2}}^{(2)}(|\psi\rangle)-\tau_{q_{1} \mid q_{3}}^{(2)}(|\psi\rangle) .
$$

Interestingly, this quantity does not depend on the focus qubit (e.g., $q_{1}$ ) that we privilege in the decomposition. Namely, $\tau_{q_{1}\left|q_{2}\right| q_{3}}^{(3)}(|\psi\rangle)=\tau_{q_{2} \mid\left(q_{3} q_{1}\right)}^{(1)}(|\psi\rangle)-\tau_{q_{2} \mid q_{3}}^{(2)}(|\psi\rangle)-\tau_{q_{2} \mid q_{1}}^{(2)}(|\psi\rangle)=$ $\tau_{q_{3} \mid\left(q_{1} q_{2}\right)}^{(1)}(|\psi\rangle)-\tau_{q_{3} \mid q_{1}}^{(2)}(|\psi\rangle)-\tau_{q_{3} \mid q_{2}}^{(2)}(|\psi\rangle)$ as well [12]. The threetangle is a full-fledged measure of the genuine tripartite entanglement of any three-qubit pure state $|\psi\rangle[1,21]$.

A generalization of the CKW inequality (1) to $n$-qubit systems was only proven by Osborne and Verstraete [16] several years after the original conjecture [12]. Denoting now by $|\psi\rangle$ a general pure state of $n$ qubits, the following holds [16],

$$
\tau_{q_{1} \mid\left(q_{2} \cdots q_{n}\right)}^{(1)}(|\psi\rangle) \geq \tau_{q_{1} \mid q_{2}}^{(2)}(|\psi\rangle)+\tau_{q_{1} \mid q_{3}}^{(2)}(|\psi\rangle)+\ldots+\tau_{q_{1} \mid q_{n}}^{(2)}(|\psi\rangle) .
$$

This means that the entanglement between $q_{1}$ and the rest is not less than the sum of the individual pairwise entanglements involving $q_{1}$ and each of the other $n-1$ qubits $q_{j}(j=2, \ldots, n)$. However, for $n>3$, the difference between left and right hand side in (4) just gives a rough indicator of all the leftover entanglement not distributed in pairwise form. Attempts to construct generalized monogamy inequalities in $n$-qubit systems have been considered [3, 17.-19], but these have not led to clear recipes to isolate the genuine $n$-partite entanglement, nor have resulted in a general sharpening of (4) for arbitrary states.

In this Letter we propose and investigate a set of sharper monogamy constraints. We raise the intuitive hypothesis that the residual in (4) is amenable to a further decomposition into individual $m$-partite contributions which involve $m=3,4, \ldots, n-1$ qubits, in all possible combinations encompassing the focus qubit $q_{1}$. Heuristically, one can expect that all of these multipartite contributions be independent, overall adding up to the global bipartite entanglement between $q_{1}$ and 
the rest of the system. This leads us to postulate a hierarchy of strong monogamy (SM) inequalities limiting the distribution of bipartite and multipartite entanglement in $n$-qubit systems, which take in general the following form

$$
\begin{gathered}
\tau_{q_{1} \mid\left(q_{2} \cdots q_{n}\right)}^{(1)}(|\psi\rangle) \geq \sum_{m=2}^{n-1} \sum_{j^{m}}\left[\tau_{q_{1}\left|q_{j_{1}^{m}}\right| \cdots \mid q_{j_{m-1}^{m}}}^{(m)}(|\psi\rangle)\right]^{\mu_{m}} \equiv \underbrace{\sum_{j=2}^{n} \tau_{q_{1} \mid q_{j}}^{(2)}(|\psi\rangle)}_{\text {2-partite }} \\
+\underbrace{\sum_{k>j=2}^{n}\left[\tau_{q_{1}\left|q_{j}\right| q_{k}}^{(3)}(|\psi\rangle)\right]^{\mu_{3}}}_{\text {3-partite }}+\ldots+\underbrace{\sum_{l=2}^{n}\left[\tau_{q_{1}\left|q_{2}\right| \cdots\left|q_{l-1}\right| q_{l+1}|\cdots| q_{n}}^{(n-1)}(|\psi\rangle)\right]^{\mu_{n-1}}}_{(n-1) \text {-partite }},
\end{gathered}
$$

where we have employed a short-hand notation, introducing the index vector $\vec{j}^{m}=\left(j_{1}^{m}, \ldots, j_{m-1}^{m}\right)$ which spans all the ordered subsets of the index set $\{2, \ldots, n\}$ with $(m-1)$ distinct elements, and we have included in general a sequence of rational exponents $\left\{\mu_{m}\right\}_{m=2}^{n-1}$, with $\mu_{2} \equiv 1$, which can regulate the weight assigned to the different $m$-partite contributions.

Our main conjecture is that inequality (5), and its variants for different choices of the focus qubit, hold simultaneously for arbitrary pure states $|\psi\rangle$ of $n$ qubits, provided one adopts a suitable definition of the $m$-partite quantities $\left\{\tau^{(m)}, \mu_{m}\right\}$. We remark that, for a given choice of the involved entanglement monotones (tangles), the expression in (5) yields a whole class of monogamy constraints, parameterized by the powers $\mu_{m}$. Any nontrivial selection of the sequence $\left\{\mu_{m}\right\}_{m=2}^{n-1}$ with $\mu_{2} \equiv$ 1 defines in fact a particular SM inequality, sharpening and generalizing the CKW one. Clearly, the verification of (5) given a set $\left\{\mu_{m}^{\star}\right\}$ implies its validity for all $\left\{\mu_{m}\right\} \geq\left\{\mu_{m}^{\star}\right\}$. For this reason, in order to establish the sharpest instance, one should aim to prove the inequalities by fixing each $\mu_{m}$ to be as small as possible, with $\mu_{m}=1 \forall m$ being the minimal choice. We will specify the adopted choices of the parameters $\mu_{m}$ in the subsequent analysis.

Interestingly, a constraint alike to (5) was shown to hold for the distribution of entanglement in permutationally-invariant continuous variable Gaussian states, leading to an operational quantification of genuine $n$-partite entanglement [20]. This gives a strong hint that a similar sharing structure should hold for entanglement in finite-dimensional systems too, although no supporting evidence was obtained prior to this work.

Setting up the notation. - Here we adopt the following prescriptions. First, we define the pure-state residual $n$-tangle $\tau^{(n)}$ as the difference between left and right hand side in (5),

$$
\tau_{q_{1}\left|q_{2}\right| \cdots \mid q_{n}}^{(n)}(|\psi\rangle):=\tau_{q_{1} \mid\left(q_{2} \cdots q_{n}\right)}^{(1)}(|\psi\rangle)-\sum_{m=2}^{n-1} \sum_{\vec{j}^{m}}\left[\tau_{q_{1}\left|q_{j_{1}^{m}}\right| \cdots \mid q_{j_{m-1}^{m}}^{m}}^{(m)}(|\psi\rangle)\right]^{\mu_{m}}
$$

In this way, the conjectured SM inequality (5) is recast into the nonnegativity of the residual, $\tau_{q_{1}\left|q_{2}\right| \cdots \mid q_{n}}^{(n)}(|\psi\rangle) \geq 0$, where the ordering of the subscripts in (6) reflects the choice of the focus qubit, which occupies the first slot (we do not expect permutation invariance for $n>3$ ). Next, we extend the residual $n$-tangle $\tau^{(n)}$ to a mixed state $\rho$ of $n$ qubits via a convenient and physically motivated convex roof procedure,

$$
\tau_{q_{1}\left|q_{2}\right| \cdots \mid q_{n}}^{(n)}(\rho):=\left[\inf _{\left\{p_{r},\left|\psi_{r}\right\rangle\right\}} \sum_{r} p_{r} \sqrt{\tau_{q_{1}\left|q_{2}\right| \cdots \mid q_{n}}^{(n)}\left(\psi_{r}\right)}\right]^{2},
$$

where the minimization is taken over all possible pure-state decompositions of the state $\rho=\sum_{r} p_{r}\left|\psi_{r}\right\rangle\left\langle\psi_{r}\right|$. For $n=3$, the definition (7) reduces to the mixed-state extension of the three-tangle $\tau^{(3)}$ as defined in [22], which is an entanglement monotone [1, 12, 21] and an invariant under stochastic local operations and classical communication (SLOCC) [3, 15, 22, 23]. For $n=2$, we recover the standard pairwise tangle, $\tau_{q_{i} \mid q_{j}}^{(2)}=C_{q_{i} \mid q_{j}}^{2}$, with the concurrence [13, 14] defined as $C_{q_{i} \mid q_{j}}=\max \left\{0, \lambda_{1}-\lambda_{2}-\lambda_{3}-\lambda_{4}\right\}$, where $\left\{\lambda_{j}\right\}$ are the squareroots of the eigenvalues (in decreasing order) of the matrix $R=\rho_{i j}\left(\sigma_{y} \otimes \sigma_{y}\right) \rho_{i j}^{*}\left(\sigma_{y} \otimes \sigma_{y}\right)$, the star denoting complex conjugation in the computational basis, $\sigma_{y}$ being the Pauli $y$ matrix, and $\rho_{i j}$ being the marginal state of qubits $q_{i}$ and $q_{j}$ obtained by partial tracing over the remaining qubits. Finally, we use Eqs. (6) - 7) to define, in a recursive way, every $m$-partite term $\tau^{(m)}$ (for $m \geq 2$ ) appearing in the $n$-qubit SM inequality (5), in terms of the corresponding residual $m$-tangle rescaled by a suitable exponent $\mu_{m}$.

Proving the SM conjecture for $n$ qubits appears in general a formidable challenge. Namely, at variance with the CKW case, the $m$-tangles defined above are not expected to enjoy a closed formula on the marginal $m$-qubit mixed states for $m \geq 3$. Nonetheless, in the following we verify the conjecture analytically on relevant multiqubit states, and we achieve significant progress on arbitrary states of four qubits $(n=4)$, for which we provide a comprehensive collection of analytical and numerical evidence in support of the SM hypothesis.

Analytical example: GHZ/W superpositions.- We begin by investigating the SM constraint (5) in its sharpest form $\left(\mu_{m}=1 \forall m\right)$, on permutationally invariant states defined as superpositions of $W$ and generalized Greenberger-HorneZeilinger (GHZ) states of $n \geq 4$ qubits,

$$
\left|\Phi_{\alpha, \beta, \gamma}^{n}\right\rangle:=\alpha\left|0^{n}\right\rangle+\beta\left|W_{n}\right\rangle+\gamma\left|1^{n}\right\rangle,
$$

with $\alpha, \beta, \gamma \in \mathbb{C},|\alpha|^{2}+|\beta|^{2}+|\gamma|^{2}=1$, where $\left|W_{n}\right\rangle=$ $\frac{1}{\sqrt{n}}\left(\left|0^{n-1} 1\right\rangle+\ldots+\left|10^{n-1}\right\rangle\right)$ is the $n$-qubit $W$ state, $\left|\Phi_{1 / \sqrt{2}, 0,1 / \sqrt{2}}^{n}\right\rangle$ is the $n$-qubit GHZ states, and $x^{n}$ denotes the string with $n$ equal symbols $x$. Noting that we can rewrite the states as $\left|\Phi_{\alpha, \beta, \gamma}^{n}\right\rangle=\left|0^{n-m}\right\rangle\left(\alpha\left|0^{m}\right\rangle+\sqrt{\frac{m}{n}} \beta\left|W_{m}\right\rangle\right)+\sqrt{\frac{n-m}{n}} \beta\left|W_{n-m}\right\rangle\left|0^{m}\right\rangle+$ $\gamma\left|1^{n-m}\right\rangle\left|1^{m}\right\rangle$, for $1 \leq m \leq n-1$, where $\left|W_{1}\right\rangle \equiv|1\rangle$, and observing in particular that for $\gamma=0$ all residual multipartite terms vanish, $\tau^{(1)}\left(\left|\Phi_{\alpha, \beta, 0}^{n}\right\rangle\right)=(n-1) \tau^{(2)}\left(\left|\Phi_{\alpha, \beta, 0}^{n}\right\rangle\right)$, we obtain the following inductive result. Assume the SM inequality (5) holds for arbitrary pure states of $m<n$ qubits, then for the $n$-qubit states $\left|\Phi_{\alpha, \beta, \gamma}^{n}\right\rangle$ one has: $\tau^{(1)}=$ $\frac{4}{n^{2}}\left[n^{2}|\alpha|^{2}|\gamma|^{2}+(n-1)|\beta|^{2}\left(|\beta|^{2}+n|\gamma|^{2}\right)\right], \tau^{(2)} \leq \frac{4|\beta|^{4}}{n^{2}}, \tau^{(n-1)} \leq$ $\frac{4}{n}|\beta|^{2}|\gamma|^{2}, \tau^{(m)}=0$ for $2<m<n-1$. Substituting these 
into Eq. 6 6, one finds: $\tau_{q_{1} \ldots \ldots q_{n}}^{(n)}\left(\left|\Phi_{\alpha, \beta, \gamma}^{n}\right\rangle\right) \geq 4|\alpha|^{2}|\gamma|^{2} \geq 0$, which proves the SM inequality (5) for the $n$-qubit states of Eq. (8). As the SM clearly holds for three-qubit states, this yields a complete analytical SM proof for generalized GHZ/W superpositions $\left|\Phi_{\alpha, \beta, \gamma}^{4}\right\rangle$ of $n=4$ qubits, which embody archetypical representatives of genuine multiparticle entanglement.

Four-qubit strong monogamy: Toolkit. - Motivated by the above result, we now analyze arbitrary pure states $|\psi\rangle$ of fourqubit systems $(n=4)$. Here, a preliminary numerical exploration reveals that the choice $\mu_{m}=1$ in (5) is too strong to hold, as it leads to negative residual four-tangles on a small subset of states [36]. Therefore, we focus on testing the SM inequality for a successive level of the hierarchy, namely we set $\mu_{m}:=m / 2(m \geq 2)$. Sticking with $q_{1}$ as focus, and according to our adopted conventions, the SM inequality (5) then specializes to (see Fig. 1 for a graphical representation)

$\tau_{q_{1} \mid\left(q_{2} q_{3} q_{4}\right)}^{(1)} \geq \tau_{q_{1} \mid q_{2}}^{(2)}+\tau_{q_{1} \mid q_{3}}^{(2)}+\tau_{q_{1} \mid q_{4}}^{(2)}+\left[\tau_{q_{1}\left|q_{2}\right| q_{3}}^{(3)}\right]^{\frac{3}{2}}+\left[\tau_{q_{1}\left|q_{3}\right| q_{4}}^{(3)}\right]^{\frac{3}{2}}+\left[\tau_{q_{1}\left|q_{2}\right| q_{4}}^{(3)}\right]^{\frac{3}{2}}$,

where we omitted the state $(|\psi\rangle)$ for brevity.

All the quantities in (9) are well defined. The bipartite terms $\tau^{(m)}$ with $m=1,2$ are all computable as described above, and the tripartite terms $\tau^{(3)}$ are to be evaluated on the reduced rank2 mixed state $\rho_{i j k}$ of qubits $q_{i}, q_{j}$, and $q_{k}$, via the prescription in Eq. (7). Let us recall that the three-tangle of three-qubit pure states $|\psi\rangle$ admits the following closed expression [12],

$$
\begin{aligned}
\tau_{q_{1}\left|q_{2}\right| q_{3}}^{(3)}(|\psi\rangle)= & 4 \mid c_{000}^{2} c_{111}^{2}+c_{001}^{2} c_{110}^{2}+c_{010}^{2} c_{101}^{2}+c_{100}^{2} c_{011}^{2} \\
& -2\left(c_{000} c_{111} c_{001} c_{110}+c_{000} c_{111} c_{010} c_{101}\right. \\
& +c_{000} c_{111} c_{100} c_{011}+c_{001} c_{110} c_{010} c_{101} \\
& \left.+c_{001} c_{110} c_{011} c_{100}+c_{100} c_{011} c_{010} c_{101}\right) \\
& +4\left(c_{000} c_{011} c_{101} c_{110}+c_{111} c_{100} c_{010} c_{001}\right) \mid
\end{aligned}
$$

where we have expanded the state $|\psi\rangle$ in the computational basis as $|\psi\rangle=\sum_{r, s, t=0}^{1} c_{r s t}|r s t\rangle$. However, to date, there is no closed formula for the three-tangle of three-qubit mixed states. The minimization in Eq. (7) has been solved only for special families of states [15, 24, 25, 29], while a semi-analytic method to determine when $\tau_{q_{i}\left|q_{j}\right| q_{k}}^{(3)}$ vanishes is generally available for rank-2 states such as $\rho_{i j k}$ [24]. We then resort to looking for tractable upper bounds to the tripartite terms [24, 2629], say $\tau_{q_{i}\left|q_{j}\right| q_{k}}^{(3) \text { up }} \geq \tau_{q_{i}\left|q_{j}\right| q_{k}}^{(3)}$. A lower bound to the residual fourtangle of Eq. (6) is then, for a four-qubit state $|\psi\rangle$,

$$
\tau_{q_{1}\left|q_{2}\right| q_{3} \mid q_{4}}^{(4) \text { low }}:=\tau_{q_{1} \mid\left(q_{2} q_{3} q_{4}\right)}^{(1)}-\sum_{j=2}^{4} \tau_{q_{1} \mid q_{j}}^{(2)}-\sum_{k>j=2}^{4}\left[\tau_{q_{1}\left|q_{j}\right| q_{k}}^{(3) \text { up }}\right]^{\frac{3}{2}}
$$

and the SM inequality may then be verified by proving that $\tau_{q_{1}\left|q_{2}\right| q_{3} \mid q_{4}} \geq 0$. We will exploit in particular the bound recently introduced by Rodriques, Datta, and Love (RDL) [28] in terms of the so-called best $W$-class approximation of three-qubit states $\rho_{i j k}$ [30]. For each rank-2 three-qubit state $\rho_{i j k} \equiv \rho$, which can be written in its spectral decomposition as $\rho=$ $\lambda|1\rangle\langle 1|+(1-\lambda)| 2\rangle\langle 2|$, one can construct an associated simplex $\mathcal{S}_{0}$ containing states with vanishing three-tangle, obtained as mixtures of (up to) four pure $W$-class states $\left|Z_{l}\right\rangle(l=1, \ldots, 4)$ [30]. The latter take the form $\left|Z_{l}\right\rangle=\left(|1\rangle+z_{l}|2\rangle\right) / \sqrt{1+\left|z_{l}\right|^{2}}$, where $z_{l} \in \mathbb{C}$ are the complex roots of the fourth-order equation $\tau^{(3)}(|1\rangle+z|2\rangle)=0$, defined via Eq. [10] [24]. If the rank-2 state $\rho$ belongs to the simplex $\mathcal{S}_{0}$, then $\tau^{(3)}(\rho)=0$. More generally, one can bound the three-tangle from above as follows. Defining the uniform mixture $\pi=\frac{1}{4} \sum_{l=1}^{4}\left|Z_{l}\right\rangle\left\langle Z_{l}\right|$, there exists a $\kappa>0$ such that $|\phi\rangle\langle\phi|:=\rho+\frac{\kappa}{\|\rho-\pi\|_{1}}(\rho-\pi)$ describes a pure three-qubit state [28], where $\|X\|_{1}=\operatorname{Tr} \sqrt{X^{\dagger} X}$ denotes the trace norm. One has then

$$
\tau^{(3)}(\rho) \leq \tau^{(3) \text { up }}(\rho):=\frac{\|\rho-\pi\|_{1}^{2}}{\||\phi\rangle\langle\phi|-\pi \|_{1}^{2}} \tau^{(3)}(|\phi\rangle),
$$

where $\tau^{(3)}(|\phi\rangle)$ can be computed from Eq. 10$\rangle$.

Four-qubit strong monogamy: Results. - For four qubits, there are infinitely many inequivalent SLOCC classes [3] (unlike the case of three qubits [21]); however, a particularly insightful classification into nine groups was derived by Verstraete et al. [22], who showed that, up to permutations of the four qubits, any pure state $|\psi\rangle$ can be obtained as

$$
|\psi\rangle=\left(A_{1} \otimes A_{2} \otimes A_{3} \otimes A_{4}\right)\left|G^{x}\right\rangle,
$$

where $\left\{A_{k}\right\} \in S L(2, \mathbb{C})$ are SLOCC operations with $\operatorname{det}\left(A_{k}\right)=$ 1 , and each $\left|G^{x}\right\rangle$ denotes a normal-form family of states, representative of the corresponding $x^{\text {th }}$ class, with $x=1, \ldots, 9$, see Table Ifor their definition; only class-1 states are generic.

We verified the proposed SM inequality (9) for the normalform representatives $\left|G^{x}\right\rangle$ of all the nine classes, by obtaining suitable analytic upper bounds to the $\tau^{(3)}$ terms in all the three-qubit marginal partitions, as presented in Table $[$ Combining these bounds with the easily computable one-tangles $\tau_{\left(q_{i} \mid q_{j} q_{k} q_{l}\right)}^{(1)}$, and with the expressions of the reduced two-tangles $\tau_{q_{i} \mid q_{j}}^{(2)}($ not reported here), we obtained lower bounds to the residual $\tau_{q_{i}\left|q_{j}\right| q_{k} \mid q_{l}}^{(4)}$ defined as in Eq. 11 , which were found to be nonnegative for all the nine families of states. These are plotted in Fig. 2(a) for some typical instances of $\left|G^{x}\right\rangle$ with $x=2, \ldots, 6$. The other cases are straightforward, in particular for $\left|G^{1}\right\rangle$ and $\left|G^{7}\right\rangle$ all the reduced three-tangles vanish, so the SM reduces to the conventional inequality (4).

We complement this collection of analytical results with a numerical exploration of arbitrary four-qubit states $|\psi\rangle$. Precisely, we generated them according to the prescription in Eq. [13, by the application of random SLOCC operations on $\left|G^{x}\right\rangle$ states with randomized parameters (a Gaussian distribution was used to generate the matrix elements of SLOCC operations on each qubit, and a uniform distribution in a bounded interval was used to generate the complex parameters in the states $\left.\left|G^{x}\right\rangle\right)$. We tested $10^{6}$ states per class, and on each state we computed the lower bound $\tau_{q_{i}\left|q_{j}\right| q_{k} \mid q_{l}}^{(4) \text { low }}$ for all four independent permutations of $\{i j k l\}$, using the semi-analytical RDL method [28] to bound the marginal three-tangles in all relevant three-qubit partitions via Eq. (12). Overall, this amounts to $3.2 \times 10^{7}$ tested data points across all the different classes (class- 9 states are excluded since for them $q_{1}$ is separable from 


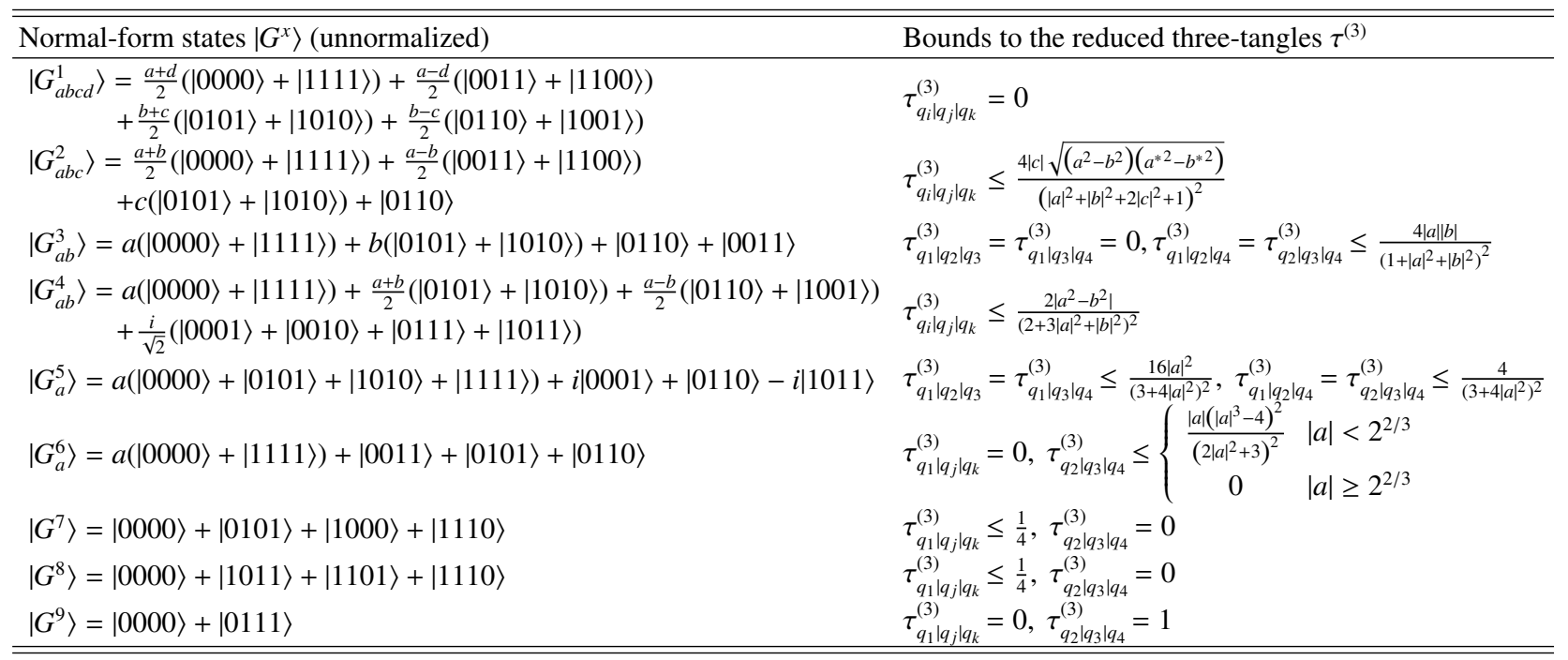

TABLE I: Normal-form representatives of the nine four-qubit SLOCC classes defined in [22], and upper bounds to the three-tangle of their marginal three-qubit partitions $q_{i}\left|q_{j}\right| q_{k}$; here $a, b, c, d$ are complex parameters with nonnegative real part.

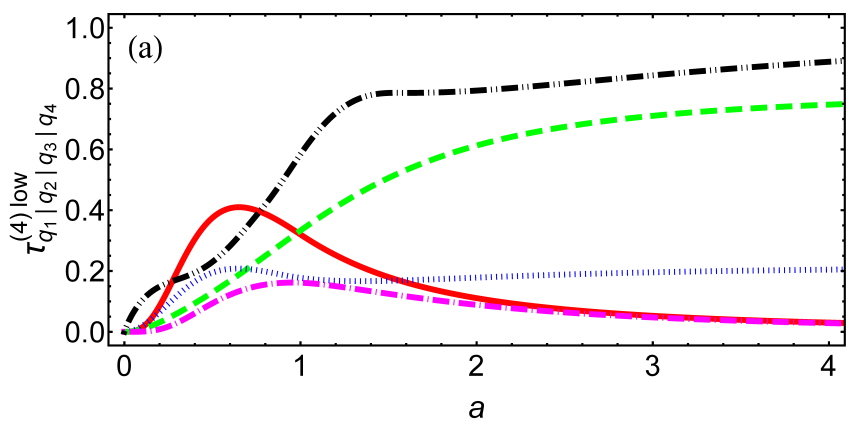

FIG. 2: (Color online) (a) Lower bound to the residual four-tangle $\tau_{q_{1}\left|q_{2}\right| q_{3} \mid q_{4}}^{(1) \operatorname{low}}$ versus the parameter $a$ (here assumed real) for the normal-form states: $\left|G_{a b c}^{2}\right\rangle$ with $b=c=a$ (red solid line), $\left|G_{a b}^{3}\right\rangle$ with $b=a / 4$ (green dashed line), $\left|G_{a b}^{4}\right\rangle$ with $b=a / 2$ (blue dotted line), $\left|G_{a}^{5}\right\rangle$ (magenta dot-dashed line), $\left|G_{a}^{6}\right\rangle$ (black dot-dot-dashed line). The residuals stay nonnegative for general choices of the parameters $a, b, c$. (b) Lower bound to the residual four-tangle $\tau_{q_{i}\left|q_{j}\right| q_{k} \mid q_{l}}^{(4)-{ }_{l}}$ versus the one-tangle $\tau_{q_{i} \mid\left(q_{j} q_{k} q_{l}\right)}^{(1)}$ for $8 \times 10^{6}$ random four-qubit pure states, with 4 partitions tested per state. Each point is gray-scaled according to the SLOCC class of the state, from $90 \%$ gray (darkest, class 1) to 20\% gray (lightest, class 8 ). The solid line is saturated by GHZ states. All the data points are above the horizontal axis, verifying the SM inequality 9 ).

the rest, so the SM constraint reduces to the CKW one for $q_{2}, q_{3}, q_{4}$ which needs no testing). As Fig.2(b) shows, no negative values of $\tau^{(4) \text { low }}$ were found, providing a strongly supportive evidence for the validity of the SM inequality 9 on arbitrary four-qubit states.

Conclusion. - We proposed and analyzed a novel class of monogamy inequalities for multiqubit entanglement, which extend and sharpen the existing ones [12,16]. We proved our $\mathrm{SM}$ relation on relevant families of states, and verified it numerically on arbitrary pure states of four qubits spanning all the different SLOCC classes of Ref. [22].

This Letter opens an avenue for further investigation. First, a closed formula for the three-tangle of rank-2 states of three qubits [15, 24, 25] could facilitate a general analytical proof of inequality (5) for $n=4$. More generally, would other entanglement measures which satisfy conventional monogamysuch as the squashed entanglement [31]) - obey SM-type inequalities too, for arbitrary multipartite states of $n$ qudits? The

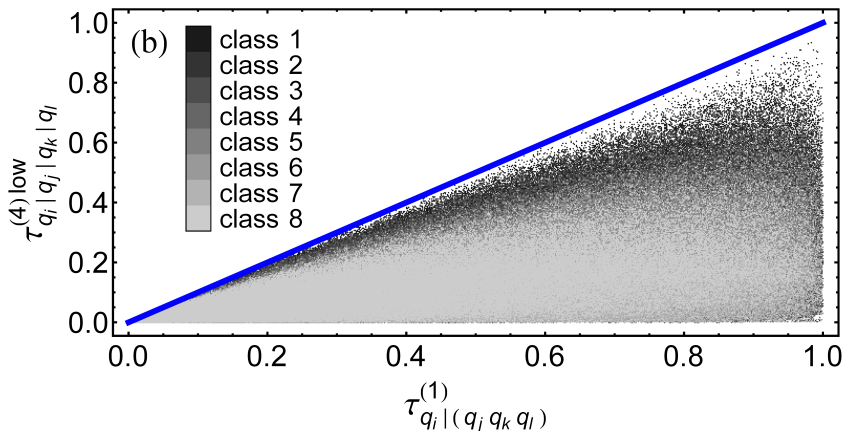

standard CKW-type monogamy [12, 16] inspired remarkable applications to quantum cryptography [1] and the characterization of quantum critical points in many-body systems [2]. This work reveals more severe limitations on the sharing of multiple forms of entanglement, and is a starting point towards a quantification of those essential features of quantum correlations, which only emerge beyond the bipartite scenario. It will be fascinating to investigate the interplay between the SM trade-off and frustration phenomena in complex quantum systems [32-34].

Acknowledgments. - We acknowledge discussions with M. Cianciaruso, N. Datta, C. Eltschka, P. Facchi, G. Florio, F. Illuminati, J. S. Kim, T. Osborne, S. Pascazio, M. Piani, J. Siewert, A. Winter, and W. K. Wootters. The authors would like to thank A. Osterloh for bringing the error in the reduced three-tangle of class-2 states $\left|G_{a b c}^{2}\right\rangle$ in the previously published version of this manuscript to their attention (see also Ref. [35]). This work has been supported by the Uni- 
versity of Nottingham, the University of Bari, the Italian National Group of Mathematical Physics (GNFM-INdAM), the Foundational Questions Institute (FQXi-RFP3-1317), and the Basic Science Research Program through the National Research Foundation of Korea funded by the Ministry of Education (NRF-2012R1A1A2003441).

[1] R. Horodecki, P. Horodecki, M. Horodecki, and K. Horodecki, Rev. Mod. Phys. 81, 865 (2009) .

[2] L. Amico, R. Fazio, A. Osterloh, and V. Vedral, Rev. Mod. Phys. 80, 517 (2008).

[3] C. Eltschka and J. Siewert, arXiv:1402.6710 (2014).

[4] B. M. Terhal, IBM J. Res. \& Dev. 48, 71 (2004).

[5] W. K. Wootters and W. H. Zurek, Nature 299, 802 (1982).

[6] G. Adesso and F. Illuminati, Int. J. Quant. Inf. 4, 383 (2006).

[7] J. Bae and A. Acin, Phys. Rev. Lett. 97, 030402 (2006); A. Kay, D. Kaszlikowski, and R. Ramanathan, Phys. Rev. Lett. 103, 050501 (2009).

[8] B. Toner and F. Verstraete, arXiv:quant-ph/0611001; B. Toner, Proc. R. Soc. A 465, 59 (2009).

[9] M. D. Reid, Phys. Rev. A 88, 062108 (2013); A. Milne, S. Jevtic, D. Jennings, H. Wiseman, and T. Rudolph, New J. Phys. 16, 083017 (2014).

[10] R. Ramanathan, A. Soeda, P. Kurzynski, and D. Kaszlikowski, Phys. Rev. Lett. 109, 050404 (2012); P. Kurzynski, A. Cabello, and D. Kaszlikowski, Phys. Rev. Lett. 112, 100401 (2014).

[11] A. Streltsov, G. Adesso, M. Piani, and D. Bruss, Phys. Rev. Lett. 109, 050503 (2012).

[12] V. Coffman, J. Kundu, and W. K. Wootters, Phys. Rev. A 61, 052306 (2000).

[13] S. Hill and W. K. Wootters, Phys. Rev. Lett. 78, 5022 (1997).

[14] W. K. Wotters, Phys. Rev. Lett. 802245 (1998).

[15] O. Viehmann, C. Eltschka, and J. Siewert, Appl. Phys. B 106, 533 (2012).

[16] T. J. Osborne and F. Verstraete, Phys. Rev. Lett. 96220503
(2006).

[17] C. Eltschka, A. Osterloh, and J. Siewert, Phys. Rev. A 80, 032313 (2009).

[18] G. Gour and N. R. Wallach, J. Math. Phys. 51, 112201 (2010).

[19] M. F. Cornelio, Phys. Rev. A 87, 032330 (2013).

[20] G. Adesso and F. Illuminati, Phys. Rev. Lett. 99, 150501 (2007); ibid., Phys. Rev. A 78, 042310 (2008).

[21] W. Dür, G. Vidal, and J. I. Cirac. Phys. Rev. A 62, 062314 (2000).

[22] F. Verstraete, J. Dehaene, B. De Moor, and H. Verschelde, Phys. Rev. A 65, 052112 (2002).

[23] F. Verstraete, J. Dehaene, and B. De Moor, Phys. Rev. A 68, 012103 (2003).

[24] R. Lohmayer, A. Osterloh, J. Siewert, and A. Uhlmann, Phys. Rev. Lett. 97, 260502 (2006).

[25] C. Eltschka, A. Osterloh, J. Siewert, and A. Uhlmann, New J. Phys. 10, 043014 (2008).

[26] T. J. Osborne, Phys. Rev. A 72, 022309 (2005).

[27] J. Zhu, S. Kais, A. Aspuru-Guzik, S. Rodriques, B. Brock, and P. J. Love, J. Chem. Phys. 137, 074112 (2012).

[28] S. Rodriques, N. Datta, and P. J. Love, Phys. Rev. A 90, 012340 (2014).

[29] C. Eltschka and J. Siewert, Phys. Rev. A 89, 022312 (2014).

[30] By $W$-class states [12, 21, 24] we refer to three-qubit pure states whose three-tangle $\tau^{(3)}$ as defined in Eq. 10 vanishes.

[31] M. Christandl and A. Winter, J. Math. Phys. 45, 829 (2003); M. Koashi and A. Winter, Phys. Rev. A 69, 022309 (2004).

[32] P. Facchi, G. Florio, G. Parisi, and S. Pascazio, Phys. Rev. A 77, 060304(R) (2008).

[33] S. M. Giampaolo, G. Adesso, and F. Illuminati, Phys. Rev. Lett. 104, 207202 (2010).

[34] S. M. Giampaolo, G. Gualdi, A. Monras, and F. Illuminati, Phys. Rev. Lett. 107, 260602 (2011).

[35] A. Osterloh, The nine ways of four qubit entanglement and their threetangle, arXiv:1512.02468 (2015).

[36] The states with negative residuals in the case $\mu_{m}=1$ were all found within the SLOCC class 4 according to the classification of [22]. 